\title{
Maladies bactériennes invasives dans le Nord du Canada, 1999 à 2018
}

\author{
Grace Huang ${ }^{1}$, Irene Martin², Raymond S Tsang², Walter H Demczuk², Gregory J Tyrrell ${ }^{3}$, \\ Y Anita Li*, Catherine Dickson'1, Francesca Reyes-Domingo', Susan G Squires ${ }^{1}$
}

\section{Résumé}

Contexte : Le programme de surveillance circumpolaire internationale assure la surveillance de cinq maladies bactériennes invasives : les pneumococcies invasives, les infections invasives à streptocoque du groupe $\mathrm{A}$, I'Haemophilus influenzae, les infections invasives à méningocoque et les streptocoques du groupe B. Les maladies bactériennes invasives ont une charge de morbidité plus élevée dans les populations nordiques que dans le reste du Canada.

Méthodologies : Afin de décrire l'épidémiologie des maladies bactériennes invasives dans le nord du Canada de 1999 à 2018, les données relatives aux pneumococcies invasives, aux infections invasives à streptocoque du groupe $\mathrm{A}$, à l'Haemophilus influenzae, aux infections invasives à méningocoque et aux streptocoques du groupe $B$ ont été extraites du programme de surveillance circumpolaire internationale et du Système canadien de surveillance des maladies à déclaration obligatoire (SSMDO) et analysées.

Résultats : Les taux d'incidence annualisés des pneumococcies invasives, des infections invasives à streptocoque du groupe $\mathrm{A}$, de l'Haemophilus influenzae, des streptocoques du groupe $B$ et des infections invasives à méningocoque étaient respectivement de $23,3,10,5$, $8,9,1,9$ et 1,1 pour 100000 habitants. L'incidence de la pneumococcie invasive, de l'infection invasive à streptocoque du groupe $\mathrm{A}$ et de l'Haemophilus influenzae de type b (Hib) était respectivement 2,8,3,2 et 8,8 fois plus élevée dans le Nord du Canada que dans le reste du pays. Les taux de maladie ont diminué de manière statistiquement significative pour la pneumococcie invasive $(\beta=-0,02)$ et ont augmenté de manière statistique pour l'infection invasive à streptocoque du groupe $A(\beta=0,08)$ et l'Haemophilus influenzae sérotype a $(\beta=0,04)$ pendant la période d'étude. Dans le Nord du Canada, les taux d'incidence annualisés des pneumococcies invasives, des infections invasives à streptocoque du groupe $A$ et de l'Haemophilus influenzae étaient statistiquement plus élevés chez les résidents autochtones que chez les résidents non autochtones. Les taux $d$ 'incidence les plus élevés ont été enregistrés dans les groupes d'âge très jeunes et plus âgés.

Conclusion : Les maladies bactériennes invasives représentent une charge de morbidité importante pour les populations nordiques du Canada. Les peuples autochtones, les enfants et les personnes âgées sont particulièrement exposés.
Cette oeuvre est mise à la disposition selon les termes de la licence internationale Creative Commons Attribution 4.0

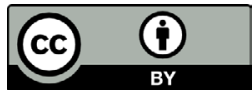

Affiliations

${ }^{1}$ Direction générale des programmes des maladies infectieuses, Agence de la santé publique du Canada, Ottawa, ON

${ }^{2}$ Laboratoire national de microbiologie, Agence de la santé publique du Canada, Winnipeg, $\mathrm{MB}$

${ }^{3}$ Department of Laboratory Medicine and Pathology, Université de l'Alberta, Edmonton, $\mathrm{AB}$

\section{*Correspondance :}

y.anita.li@phac-aspc.gc.ca

Citation proposée : Huang G, Martin I, Tsang RS, Demczuk WH, Tyrrell GJ, Li YA, Dickson C, Reyes-Domingo F, Squires SG. Maladies bactériennes invasives dans le Nord du Canada, 1999 à 2018. Relevé des maladies transmissibles au Canada 2021;47(11):545-54. https://doi.org/10.14745/ccdr.v47i11a09f

Mots-clés : Santé des populations autochtones, surveillance, Haemophilus influenzae, streptocoque, méningococcie, pneumococcie, vaccin, épidémiologie

\section{Introduction}

Le programme de surveillance circumpolaire internationale est un réseau de surveillance des maladies bactériennes invasives basé sur la population des pays ayant des régions circumpolaires (1). Le programme de surveillance circumpolaire internationale assure la surveillance des maladies bactériennes invasives causées par le Streptococcus pneumoniae (pneumococcie invasive), l'Haemophilus influenzae (Haemophilus influenzae invasif), le Streptococcus pyogenes (infection invasive à streptocoque du groupe A), le Neisseria meningitidis (infections invasives à méningocoque) et Streptococcus agalactiae 
(streptocoque du groupe B). Les taux de maladies causées par ces agents pathogènes sont élevés chez les populations autochtones des pays ayant des régions arctiques (2-7).

Le programme de surveillance circumpolaire internationale a commencé à surveiller la pneumococcie invasive au Canada en 1999 et s'est étendu à la surveillance des quatre autres maladies en 2000. Six régions canadiennes participent au programme de surveillance circumpolaire internationale : Le Nunavut, les Territoires du Nord-Ouest, le Yukon, le Nord du Labrador et deux régions du Nord du Québec, le Nunavik et le territoire des Cris. Le programme de surveillance circumpolaire internationale comprend également deux laboratoires de référence, le Laboratoire national de microbiologie et le Laboratoire de santé publique du Québec, ainsi qu'un réseau de laboratoires régionaux répartis dans tout le Canada qui dessert les régions du Nord. Jusqu'en 2009, le Centre national des streptocoques a participé au programme de surveillance circumpolaire internationale en tant que laboratoire de référence.

En 2018, la population canadienne du programme de surveillance circumpolaire internationale était estimée à 168090 personnes, soit 0,45\% de la population intercensitaire nationale totale de 37 millions de personnes. Si les autochtones représentent $4,9 \%$ de la population canadienne totale, ils constituent environ $60 \%$ de la population du nord du Canada (8).

Le Comité consultatif national de l'immunisation (CCNI) fournit des recommandations nationales en matière de vaccins pour la pneumococcie invasive, l' $H$. influenzae et l'infection invasive à méningocoque; toutefois, l'adoption de ces recommandations relève de la compétence des provinces et des territoires. Le vaccin contre $H$. influenzae sérotype $b$, le vaccin conjugué contre le méningocoque de sérogroupe $C$ (Men-C) pour les nourrissons et le vaccin Men-C-ACYW pour les jeunes adolescents font partie des programmes de vaccination systématique des enfants depuis 1997, 2007 et 2011, respectivement (9). Dans le cadre du calendrier de vaccination systématique des enfants, les six régions du Nord ont mis en œuvre le vaccin conjugué contre le pneumocoque 7-valent (Pneu-C-7) pour les enfants de moins de deux ans entre 2002 et 2007, remplaçant le Pneu-C-7 par le vaccin conjugué contre le pneumocoque 10-valent (Pneu-C-10) en 2010 (10), puis par le vaccin conjugué contre le pneumocoque 13-valent (Pneu-C-13) en 2011 (selon une enquête envoyée aux membres du groupe de travail sur les maladies bactériennes invasives de surveillance circumpolaire internationale). Au milieu de l'année 2018, le territoire des Cris et le Nunavik sont passés à un calendrier de vaccination à quatre doses de Pneu-C-10 et Pneu-C-13 (3+1) (communication personnelle, membres du groupe de travail sur les maladies bactériennes invasives du programme de SCl, 2019). Le CCNI recommande que le vaccin polysaccharidique contre le pneumocoque 23-valent (Pneu-P-23) soit administré aux personnes âgées de deux ans ou plus présentant un risque élevé de pneumococcie invasive, ainsi qu'aux adultes de 65 ans et plus (11). Les enfants et les adultes à haut risque sont ceux qui présentent certaines maladies chroniques, des états immunodéprimants ou une asplénie fonctionnelle ou anatomique (11). Les recommandations du Guide canadien d'immunisation concernant le Pneu-P-23 ont été appliquées dans les six régions, avec des différences mineures quant à l'âge d'administration du vaccin dans certaines régions (11-17).

L'objectif de ce rapport est de décrire l'épidémiologie des maladies bactériennes invasives dans le Nord du Canada au cours des 20 premières années du programme de surveillance circumpolaire internationale (1999 à 2018) et de comparer leurs taux d'incidence à ceux du reste du Canada. $\mathrm{Ce}$ rapport comprend les données de surveillance circumpolaire internationale qui ont été publiées précédemment (1999 à 2013) $(5,7)$. Ces données ont été incluses dans le présent document afin de fournir une analyse complète des maladies bactériennes invasives au cours des 20 premières années du programme surveillance circumpolaire internationale.

\section{Méthodes}

\section{Données épidémiologiques}

Les données ont été extraites du programme de surveillance circumpolaire internationale (1999 à 2018) et du Système canadien de surveillance des maladies à déclaration obligatoire (SSMDO, 2000-2018). Les données du SSMDO antérieures à 2000 n'étaient pas disponibles. Les données du SSMDO pour $l^{\prime} H$. influenzae non sérotype $b$ invasif n'ont pas été incluses car seuls certaines provinces et certains territoires les ont rapportées pour certaines années. Les données du SSMDO pour le streptocoque du groupe B n'ont pas non plus été incluses parce que seuls les streptocoques du groupe B des nouveaunés sont rapportés au niveau national alors que la surveillance circumpolaire internationale effectue une surveillance sur tous les cas de streptocoques du groupe $B$. Les cas répondant à la définition nationale de cas ont été inclus (18). Comme il n'existe pas de définition nationale de cas pour le streptocoque du groupe $B$ pour la population générale, la définition de cas de la surveillance circumpolaire internationale étend la définition de cas nationale pour le streptocoque du groupe B du nouveau-né aux personnes de tous les âges dans le Nord du Canada. Les données comprennent des renseignements démographiques non nominatifs, des renseignements cliniques, la gravité, les résultats, les conditions sous-jacentes et l'historique des vaccinations.

\section{Données de laboratoire}

Les isolats de $S$. pneumoniae ont été sérotypés par la réaction de Quellung avec des antisérums commerciaux de bassin, de groupe, de type et de facteur (Statens Serum Institut, Copenhague, Danemark) (19). Les isolats de $S$. pyogenes ont été identifiés en utilisant la $\beta$-hémolyse sur gélose au sang de mouton, la sensibilité à la bacitracine et le test de la pyrrolidonyle arylamidase. Le sérotypage $M$ des isolats invasifs de S. pyogenes de 1999 à septembre 2006 a été réalisé à 
I'aide d'un protocole de typage sérologique décrit par Tyrrell et al. (20). D'octobre 2006 à 2018, le typage emm moléculaire des isolats invasifs de $S$. pyogenes a été réalisé à l'aide de tests de réaction en chaîne par polymérase ( $P C R$ ) et de séquençage de l'ADN conformément au protocole des Centers for Disease Control and Prevention (CDC); la base de données de séquences emm de $S$. pyogenes invasif des CDC a été recherchée pour la désignation du type emm à l'aide de l'outil de recherche d'alignement local de base (BLAST) $(21,22)$. Les isolats de S. agalactiae ont été sérotypés par agglutination de particules de latex (SSI Diagnostica; Statens Serum Institute, Copenhague, Danemark) comme décrit par Slotved et al. (23). H. influenzae a été identifié à l'aide de la morphologie de la coloration de Gram et des tests biochimiques standard (24). Le test d'agglutination bactérienne sur lame, utilisant des antisérums commerciaux (Difco, Becton Dickinson, Mississauga, Ontario), a été utilisé pour le sérotypage, les résultats étant confirmés par PCR (25). N. meningitidis a été identifié par des tests biochimiques standards, le sérogroupe étant déterminé par des tests d'agglutination bactérienne utilisant des antisérums produits en interne contre les 12 sérogroupes différents (26).

Les susceptibilités antimicrobiennes phénotypiques des isolats canadiens ont été déterminées à l'aide de panels de microdilution en bouillon Sensititre STP6F (Thermo Fisher, États-Unis). Les interprétations résistantes, intermédiaires ou sensibles de la concentration minimale inhibitrice pour l'érythromycine, la clindamycine, la pénicilline, le céfépime, le céfotaxime, la ceftriaxone, le méropénème, le triméthoprime/ sulfaméthoxazole et la tétracycline ont été déterminées selon les directives du Clinical Laboratory Standards Institute (27).

\section{Données sur la population}

Les estimations pour la population ont été basées sur les estimations postcensitaires finales du 1er juillet 2016 (8). Les populations autochtones du Nord du Canada ont été estimées à partir des recensements de 1996, 2001, 2006, 2011 et 2016 (8,28-31). La proportion de la population autochtone pour une année de recensement donnée a été utilisée pour estimer la population autochtone pour les années jusqu'au prochain recensement.

\section{Analyse}

La méthode directe a été utilisée pour calculer les taux normalisés selon l'âge en multipliant les taux spécifiques à l'âge par les poids de la population générale canadienne de 2011 (32). Les intervalles de confiance (IC 95 \%) des taux standardisés pour l'âge ont été calculés en utilisant la méthode basée sur la distribution gamma (33). Les tests exacts de Fisher à deux intervalles et le test du chi carré ont été utilisés pour comparer les proportions. La régression de Poisson et la régression binomiale négative ont été utilisées pour estimer les tendances de la maladie. Le test exact de Poisson a été utilisé pour comparer les taux. La signification statistique a été fixée à $p<0,05$.

Les analyses ont été réalisées à l'aide d'Excel 2016 (Microsoft Corp., Redmond, Washington, États-Unis), SAS Enterprise Guide 7.1 (SAS Institute Inc., Cary, Caroline du Nord, États-Unis) et $R$ version 3.6.2 (R Foundation for Statistical Computing, Vienne, Autriche).

Les taux d'incidence des cas néonatals de streptocoques du groupe $B$ n'ont pas été calculés car les estimations annuelles des naissances vivantes pour les régions du Nord n'étaient pas disponibles pour ce rapport. Les taux standardisés par âge et par sexe pour l'infection invasive à méningocoque et le streptocoque du groupe $B$ n'ont pas été calculés en raison des faibles effectifs. Le streptocoque du groupe $B$ ne peut pas être déclaré dans la province de Québec, et par conséquent les territoires Cris et le Nunavik ne sont pas inclus dans les analyses.

\section{Résultats}

\section{Vue d'ensemble}

De 1999 à 2018, un total de 692 cas de pneumococcies invasives ont été signalés dans le Nord du Canada. De 2000 à 2018, un total de 311 cas d'infections invasives à streptocoque du groupe A, 258 cas d' $H$. influenzae, 44 cas de streptocoques du groupe $B$ et 31 cas d'infections invasives à méningocoque ont été signalés dans la même région. Les renseignements démographiques pour chaque maladie sont présentés dans le tableau 1, et l'incidence globale pour chaque maladie de 1999 à 2018 est présentée dans la figure 1.

\section{Tableau 1 : Caractérisation des maladies dans le nord du Canada par le taux d'incidence brut, les données démographiques, les décès et les hospitalisations, 1999-2000 à 2018}

\begin{tabular}{|c|c|c|c|c|c|c|c|c|c|c|c|c|c|c|c|}
\hline \multirow{3}{*}{ Maladie } & \multirow{3}{*}{$\mathbf{N}$} & \multirow{3}{*}{$\begin{array}{c}\text { Taux } \\
\text { d'incidence } \\
\text { brut pour } \\
100000 \\
\text { habitants }\end{array}$} & \multirow{3}{*}{$\begin{array}{c}\text { Âge } \\
\text { médian, } \\
\text { années }\end{array}$} & \multirow{3}{*}{$\begin{array}{c}\text { Tranche } \\
\text { d'âge }\end{array}$} & \multicolumn{3}{|c|}{$\begin{array}{l}\text { Taux d'incidence annualisé pour } 100 \\
000 \text { habitants par groupe ethnique }\end{array}$} & \multicolumn{4}{|c|}{ Hospitalisation } & \multicolumn{4}{|c|}{ Fatalitéa } \\
\hline & & & & & \multirow{2}{*}{$\begin{array}{c}\text { Groupe } \\
\text { ethnique } \\
\text { autochtone }\end{array}$} & \multirow{2}{*}{$\begin{array}{c}\text { Groupe } \\
\text { ethnique } \\
\text { non } \\
\text { autochtone }\end{array}$} & \multirow{2}{*}{$\begin{array}{c}\text { Différence } \\
\text { entre les } \\
\text { groupes } \\
\text { ethniques, } \\
\text { valeur } p\end{array}$} & \multicolumn{2}{|c|}{ Cas } & \multicolumn{2}{|c|}{$\begin{array}{c}\text { Rapport du taux } \\
\text { d'incidence }\end{array}$} & \multicolumn{2}{|c|}{ Décès } & \multicolumn{2}{|c|}{$\begin{array}{c}\text { Rapport du taux de } \\
\text { mortalité }\end{array}$} \\
\hline & & & & & & & & $\mathbf{N}$ & $\%$ & Homme:femme & Valeur $p$ & $\mathbf{N}$ & $\%$ & Homme:femme & Valeur $p$ \\
\hline $\mathrm{PI}$ & 692 & 23,3 & 37 & 0-98 & 31,3 & 7,0 & $<0,0001^{b}$ & 585 & 84,5 & 1,3 & $0,006^{b}$ & 59 & 8,5 & 1,3 & 0,3 \\
\hline IISGA & 311 & 10,5 & 50 & $0-98$ & 14,8 & 2,7 & $<0,0001^{b}$ & 273 & 87,8 & 1,3 & $0,03^{b}$ & 30 & 9,6 & 1,2 & 0,6 \\
\hline $\mathrm{Hi}$ & 258 & 8,9 & 1 & $0-93$ & 13,1 & 0,9 & $<0,0001^{b}$ & 222 & 86,0 & 1,1 & 0,5 & 19 & 7,4 & 1,6 & 0,3 \\
\hline SGB & 44 & 1,9 & 32,5 & $0-88$ & 1,8 & 1,3 & 0,3 & 41 & 93,2 & 0,9 & 0,7 & 0 & 0 & & $-c$ \\
\hline IIM & 31 & 1,1 & 1 & $0-56$ & 1,4 & 0,6 & 0,06 & 28 & 90,3 & 0,7 & 0,4 & 4 & 12,9 & Tous des hommes & 0,06 \\
\hline
\end{tabular}

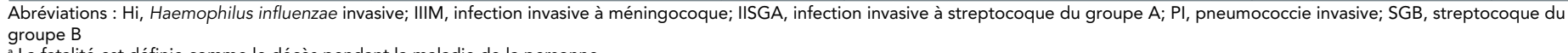

a La fatalité est définie comme le décès pendant la maladie de la personne

b Statistiquement significatif

Aucun décès n'a été signalé, ni chez les hommes ni chez les femmes 
Figure 1 : Taux d'incidence brute globale des maladies bactériennes invasives dans les régions de surveillance circumpolaire internationale, par maladie et par année, 1999 à 2018

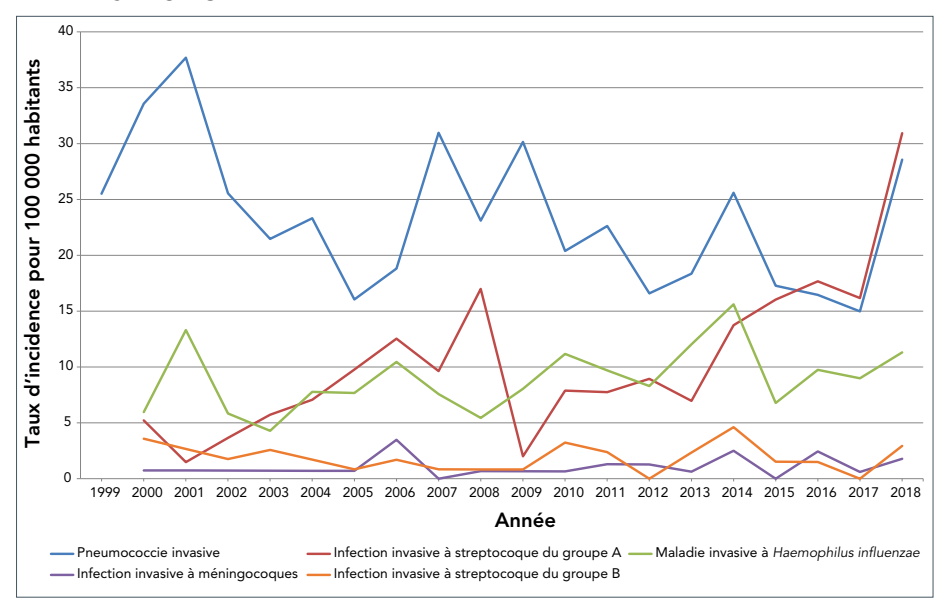

\section{Pneumococcie invasive}

Les analyses de régression ont révélé une tendance globale significative à la baisse du taux $d^{\prime}$ incidence des pneumococcies invasives (pour 100000 habitants) au fil du temps ( $\beta=-0,02$; $p=0,01$ ), mais une forte augmentation de $92 \%$ du nombre de cas signalés a été observée entre 2017 et 2018. Les taux d'incidence standardisés pour l'âge chez les hommes $(28,26$; IC : $25,30-31,53)$ et les femmes $(23,86$; IC: $20,77-27,36)$ ne différaient pas significativement. Le taux d'incidence (pour 100000 habitants) était le plus élevé chez les nourrissons de moins d'un an $(146,78 ;$ IC : 117,59-183,23), les enfants de 1 à 4 ans $(49,80$; IC : $41,13-60,29)$ et les adultes de 60 ans et plus (51,07; IC : 43,22-60,32). La différence des taux d'incidence annuels moyens entre les personnes d'origine autochtone et non autochtone était significative dans les régions du nord (tableau 1). Le taux d'incidence standardisé pour l'âge des pneumococcies invasives dans les régions nordiques était 2,8 fois plus élevé que dans le reste du Canada (tableau 2).

Les sérotypes du Pneu-C-7 ( $\beta=-0,19 ; p<0,0001)$ et les sérotypes spécifiques du Pneu-C-10 ( $\beta=-0,01 ; p<0,0001)$ ont diminué de manière significative entre 1999 et 2018 (figure 2). Les sérotypes spécifiques du Pneu-P-23 $(\beta=0,06 ; p<0,0001)$ et les sérotypes non vaccinaux ont augmenté de manière significative entre 1999 et $2018(\beta=0,04 ; p<0,05)$. II n'y a pas eu de changement statistiquement significatif des sérotypes spécifiques du Pneu-C-13 entre 1999 et 2018. Parmi les cas pour lesquels des données sur les sérotypes étaient disponibles et qui ont été collectés entre 1999 et 2010, les sérotypes 1 (18\%), $8(11 \%)$ et $14(7 \%)$ étaient les plus fréquents. Après 2010, les sérotypes les plus fréquents étaient $7 F(12 \%), 22 F(9 \%)$, $10 \mathrm{~A}(9 \%)$ et $9 \mathrm{~N}(8 \%)$.
Tableau 2 : Taux d'incidence standardisés pour l'âge (pour 100000 habitants) des maladies bactériennes invasives au Canada, par maladie, région et année, 2000-2001 à 2018a

\begin{tabular}{|l|r|r|r|r|}
\multirow{2}{*}{ Maladie } & \multicolumn{3}{|c|}{ Taux d'incidence standardisés pour l'âge } \\
\cline { 2 - 5 } & \multicolumn{2}{|c|}{ Régions du nord } & \multicolumn{2}{c|}{ Reste du Canada } \\
\cline { 2 - 5 } & $95 \%$ & \multicolumn{1}{c|}{ Cl } & $95 \%$ & \multicolumn{1}{c|}{ Cl } \\
\hline PI $^{\mathrm{b}}$ & 25,68 & $23,45-28,09^{c}$ & 9,13 & $9,05-9,21^{c}$ \\
\hline IISGA & 14,16 & $12,31-15,86^{c}$ & 4,45 & $4,40-4,50^{c}$ \\
\hline Hib $^{\mathrm{d}}$ & 0,70 & $0,45-1,13^{\mathrm{c}}$ & 0,08 & $0,08-0,09^{c}$ \\
\hline IIM & 0,75 & $0,51-1,17$ & 0,52 & $0,51-0,54$ \\
\hline
\end{tabular}

Abréviations : IC, intervalle de confiance; Hib, Haemophilus influenzae sérotype b; IIM, infection invasive à méningocoque; IISGA, infection invasive à streptocoque du groupe $A$; $\mathrm{Pl}$, pneumococcie invasive; $\mathrm{SCl}$, Surveillance circumpolaire internationale

a Un cas de pneumococcie invasive et un cas d'infections invasives à streptocoque du groupe $\mathrm{A}$ avec âge manquant ont été exclus du calcul du taux d'incidence de surveillance circumpolaire internationale

b Les taux d'incidence normalisés selon l'âge pour les pneumococcies invasives ne comprennent pas les données pour 1999-2000, lorsque les données n'étaient pas disponibles ou n'ont pas été déclarées par toutes les provinces

'Comme les IC ne se chevauchent pas, les taux d'incidence normalisés selon l'âge pour la pneumococcie invasive, l'infection invasive à streptocoque du groupe $\mathrm{A}$ et $\mathrm{I}^{\prime} \mathrm{H}$. influenzae sérotype b sont significativement différents entre les régions du Nord et le reste du Canada des comparaisons des taux d'incidence normalisés selon l'âge pour Haemophilus influenzae de sérotype non-b (sérotypes a-f et non typables) entre le nord du Canada et le reste du Canada n'ont pas été effectuées, car ces données ne sont pas disponibles pour toutes les provinces

Figure 2 : Distribution des sérotypes de pneumococcies invasives dans le nord du Canada, par année et par sérotype de vaccina, 1999 à 2018

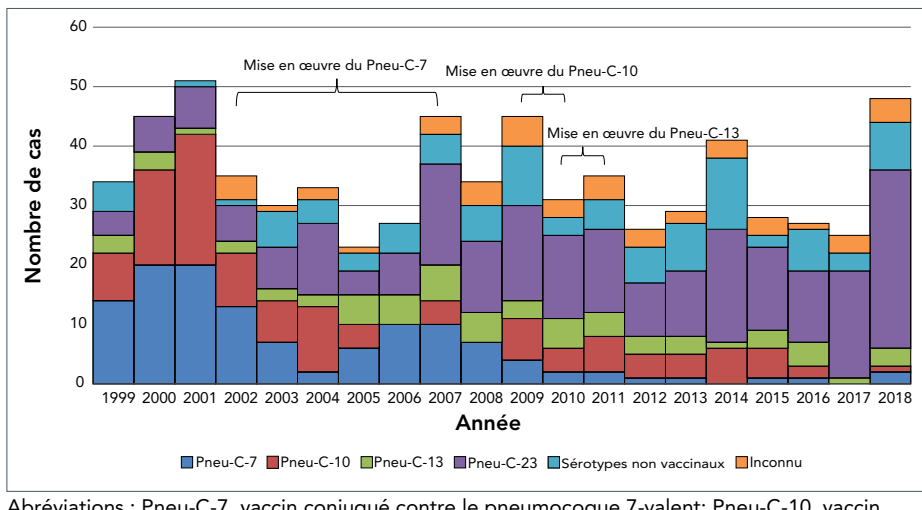

Abréviations : Pneu-C-7, vaccin conjugué contre le pneumocoque 7-valent; Pneu-C-10, vaccin conjugué contre le pneumocoque 10-valent; Pneu-C-13, vaccin conjugué contre le pneumocoque 13-valent; Pneu-P-23, vaccin polysaccharidique contre le pneumocoque 23 -valent

a Aux fins de ce graphique, le Pneu-C-7 comprend tous les sérotypes couverts par le vaccin Pneu-C-7, le Pneu-C-10 comprend les trois sérotypes supplémentaires non couverts par le vaccin Pneu-C-7, le Pneu-C-13 comprend les trois sérotypes supplémentaires non couverts par le vaccin Pneu-C-10 et le Pneu-P-23 comprend tous les sérotypes non couverts par I'un des vaccins conjugués

Toutes les régions de surveillance circumpolaire internationale avaient mis en œuvre le calendrier vaccinal du Pneu-C-13

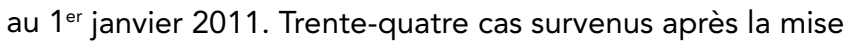
en œuvre du Pneu-C-13 ont été vaccinés en fonction de l'âge avec au moins une dose et sérotypés. Les personnes ont été vaccinées en fonction de leur âge sur la base de la recommandation nationale minimale de trois doses. Parmi ces 34 cas, trois cas ( $9 \%$ ) présentaient une infection post-vaccinale. Il y a infection post-vaccinale si le sérotype du cas est l'un des sérotypes couverts par le vaccin administré. Deux de ces cas 
étaient du sérotype 19A et un du sérotype 3. Sur les 55 cas vaccinés et sérotypés par le Pneu-P-23, 46 cas (84 \%) présentaient une infection post-vaccinale.

Sur les 670 cas pour lesquels on disposait de renseignements sur les manifestations cliniques, la bactériémie ( $n=577 ; 86,1 \%$ ) et la pneumonie ( $n=442 ; 66,0 \%$ ) étaient les manifestations les plus fréquemment rapportées. Sur les 692 cas de pneumococcie invasive, 585 (85\%) ont été hospitalisés.

Sur les 649 cas pour lesquels on disposait de renseignements sur le décès, 59 étaient mortels (9,1\%). La majorité des cas mortels étaient âgés de 60 ans et plus ( $n=24 ; 41 \%$ ), suivis par ceux âgés de 40 à 59 ans $(n=20 ; 34 \%)$. Les personnes de ces deux groupes d'âge présentaient un risque de décès significativement plus élevé que ceux des groupes d'âge plus jeunes (taux de létalité $[T L]=14,2 \%$ contre $4,4 \% ; p<0,0001)$. Le taux de létalité ne varie pas entre les autochtones et les non-autochtones $(9,5 \%$ contre $6,1 \% ; p=0,31)$. Parmi les 53 cas mortels pour lesquels on disposait de renseignements sur le sérotype, $45 \%$ étaient des sérotypes spécifiques du Pneu-P-23 non couverts par le Pneu-C-13. Les six principaux sérotypes signalés étaient les suivants : $20,10 \mathrm{~A}, 15 \mathrm{C}, 22 \mathrm{~F}, 15 \mathrm{~A}$ et 3 .

Lorsque les taux de mortalité et d'hospitalisation ont été comparés chez les hommes et les femmes, l'hospitalisation était significativement plus élevée chez les hommes (tableau 1).

La sensibilité aux antimicrobiens a été examinée pour les isolats de pneumococcie invasive pour lesquels des données étaient disponibles (tableau 3).

\section{Infection invasive à streptocoque du groupe $\mathrm{A}$}

Le taux d'incidence $(\beta=0,08 ; p<0,0001)$ de l'infection invasive à streptocoque du groupe $A$ (pour 100000 habitants) a augmenté de manière significative entre 2000 et 2018 . Les taux d'incidence standardisés pour l'âge chez les hommes $(16,07 ;$ IC : 13,65-18,84) et les femmes $(11,83$; IC : 9,58-14,52) ne différaient pas de manière significative. Le taux d'incidence (pour 100000 habitants) était le plus élevé chez les nourrissons de moins d'un an $(51,25$; IC : 34,90-75,27) et les adultes de 60 ans et plus $(36,64 ;$ IC : 30,00-44,75). La différence des taux $d$ 'incidence annuels moyens entre les personnes d'origine autochtone et non autochtone était significative dans les régions du nord (tableau 1). Le taux d'incidence normalisé selon l'âge de I'infection invasive à streptocoque du groupe $A$ dans les régions nordiques était 3,2 fois plus élevé que dans le reste du Canada (tableau 2).

Sur les 311 cas d'infections invasives à streptocoque du groupe A, 249 ont été sérotypés. Les types d'emm les plus fréquents étaient emm 1 avec 28 cas (11,2\%), emm83 avec 17 cas $(6,8 \%)$, emm3 avec 15 cas $(6,0 \%)$ et emm11 et emm59 avec 14 cas chacun (5,6\%) (figure 3 ). Le type d'emm prédominant a varié d'une année à l'autre et, si l'emm1 était en circulation la plupart
Tableau 3 : Proportion d'isolats de pneumococcie invasive et d'infections invasives à streptocoque du groupe A sensibles aux antibiotiques, 1999-2000 à $2018^{\mathrm{a}}$

\begin{tabular}{|c|c|c|}
\hline Antibiotique & $\begin{array}{c}\text { Proportion } \\
\text { d'isolats PI } \\
\text { sensibles, en \% }\end{array}$ & $\begin{array}{c}\text { Proportion } \\
\text { d'isolats IISGA } \\
\text { sensibles, en \% }\end{array}$ \\
\hline Ampicilline & 100 & 100 \\
\hline Céfotaxime & 100 & 100 \\
\hline Ceftriaxone & 98,3 & 100 \\
\hline Céfuroxime & 100 & s.o. \\
\hline Chloramphénicol & 99,8 & 98,1 \\
\hline Clindamycine & 97,3 & 90,0 \\
\hline Érythromycine & 92,4 & 81,4 \\
\hline Lévofloxacine & 99,8 & 98,9 \\
\hline Ofloxacine & 99,4 & 100 \\
\hline Oxacilline & 97,6 & 100 \\
\hline Pénicilline & 96,1 & 100 \\
\hline Rifampicine & 100 & 100 \\
\hline Tétracycline & 93,3 & 100 \\
\hline $\begin{array}{l}\text { Sulfaméthoxazole/ } \\
\text { Triméthoprime }\end{array}$ & 92,9 & 76,2 \\
\hline Vancomycine & 100 & 100 \\
\hline
\end{tabular}

des années, il n'était pas toujours la souche la plus courante pour une année donnée. La souche la plus courante en 2017 et 2018 était emm11.

Figure 3 : Répartition des cas d'infection invasive à streptocoque du groupe A par type d'emm dans le Nord du Canada, 2014 à 2018

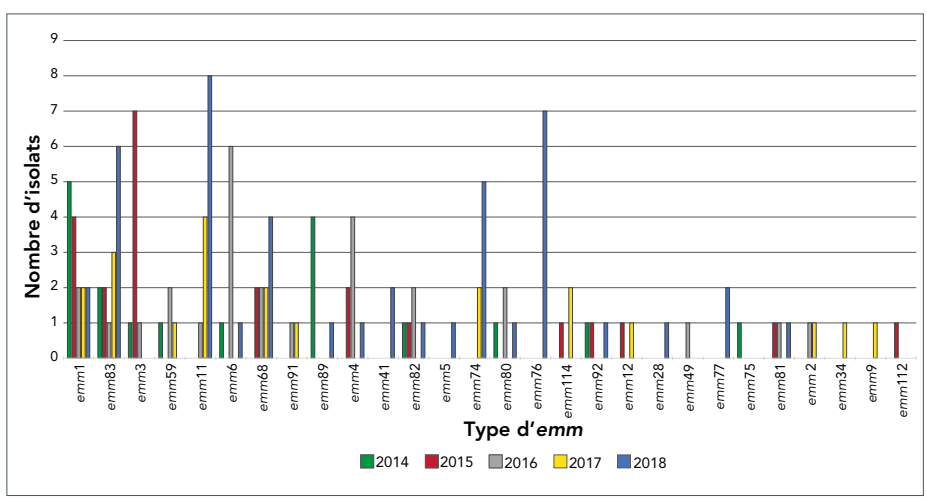

Sur les 307 cas pour lesquels on disposait de renseignements sur les manifestations cliniques, la bactériémie ( $n=196 ; 63,8 \%$ ) et la cellulite ( $n=96 ; 31,3 \%$ ) étaient les manifestations les plus fréquemment rapportées, et 273 (88,9\%) cas ont été hospitalisés. Sur les 296 cas pour lesquels on dispose de 
renseignements sur les résultats, 30 décès (TL : $10 \%$ ) ont été signalés. Lorsque les taux de mortalité et d'hospitalisation ont été comparés chez les hommes et les femmes, I'hospitalisation était significativement plus élevée chez les hommes (tableau 1). Les types d'emm présentant les taux de mortalité les plus élevés sont emm87 (50,0\%) et emm2 (50,0\%), suivis d'emm3 (33,3\%) et d'emm75 (33,3\%).

La sensibilité aux antimicrobiens a été examinée pour les isolats d'infections invasives à streptocoque du groupe A pour lesquels des données étaient disponibles (tableau 3).

\section{Maladie invasive à Haemophilus influenzae}

II n'y a pas eu de changement significatif $(\beta=0,02 ; p=0,06)$ dans le taux d'incidence de l' $H$. influenzae (pour 100000 habitants) entre 2000 et 2018 . Les taux d'incidence standardisés pour l'âge chez les hommes $(7,84 ;$ IC : 6,44-9,54) et les femmes $(6,85$; IC :5,44-8,63) ne différaient pas de manière significative. Le taux d'incidence (pour 100000 habitants) était le plus élevé chez les nourrissons de moins d'un an $(195,15 ;$ IC : 160,29-237,59), les enfants de 1 à 4 ans $(39,90$; IC : 23,05-49,67) et les adultes de 60 ans et plus $(9,54$; IC : 6,45-14,12). La différence des taux d'incidence annuels moyens entre les personnes d'origine autochtone et non autochtone était significative dans les régions du nord (tableau 1).

$H$. influenzae à sérotype a représentait $60,5 \%$ des cas $(n=156)$, les souches non typables représentaient $17,8 \%$ $(n=46), H$. influenzae sérotype b représentait 11,2\% $(n=29)$, le sérotype $f$ représentait $4,7 \%(n=12)$, les sérotypes c et d représentaient chacun $1,6 \%(n=4)$, le sérotype e représentait $0,4 \%(n=1)$ et $2,7 \%(n=6)$ avaient un sérotype inconnu (figure 4). Les taux $d^{\prime} H$. influenzae à sérotype a ont augmenté de manière significative entre 2000 et $2018(\beta=0,04$; $p=0,01)$, et $69,2 \%(n=108)$ des cas $d^{\prime} H$. influenzae à sérotype a concernaient des enfants de moins de deux ans. Le taux $d$ 'incidence de $H$. influenzae sérotype b standardisé pour l'âge dans les régions nordiques était 8,8 fois plus élevé que dans le reste du Canada (tableau 2).

II n'y a pas eu de changement significatif dans les taux de $H$. influenzae sérotype b entre 2000 et $2018(\beta=-0,01 ; p=0,7)$. Parmi les 29 cas de $H$. influenzae sérotype $b$, trois étaient des adultes et 26 des enfants âgés de trois ans ou moins. Sur les 29 cas de $H$. influenzae sérotype b, 14 (48\%) étaient vaccinés en fonction de l'âge, 10 (35\%) n'étaient pas vaccinés ou n'étaient pas à jour de leurs vaccinations, trois (10\%) n'étaient pas admissibles à la vaccination en fonction de l'âge et deux (7 \%) avaient un historique vaccinal inconnu.

Sur les 246 cas pour lesquels on disposait de renseignements sur les manifestations cliniques, la bactériémie ( $n=201 ; 81,7 \%$ ) et la pneumonie $(n=87 ; 35,4 \%$ ) étaient les manifestations les plus fréquemment rapportées. Sur les 258 cas d'H. influenzae,
Figure 4 : Distribution des sérotypes invasifs d'Haemophilus influenzae dans le nord du Canada par année, 2000 à 2018

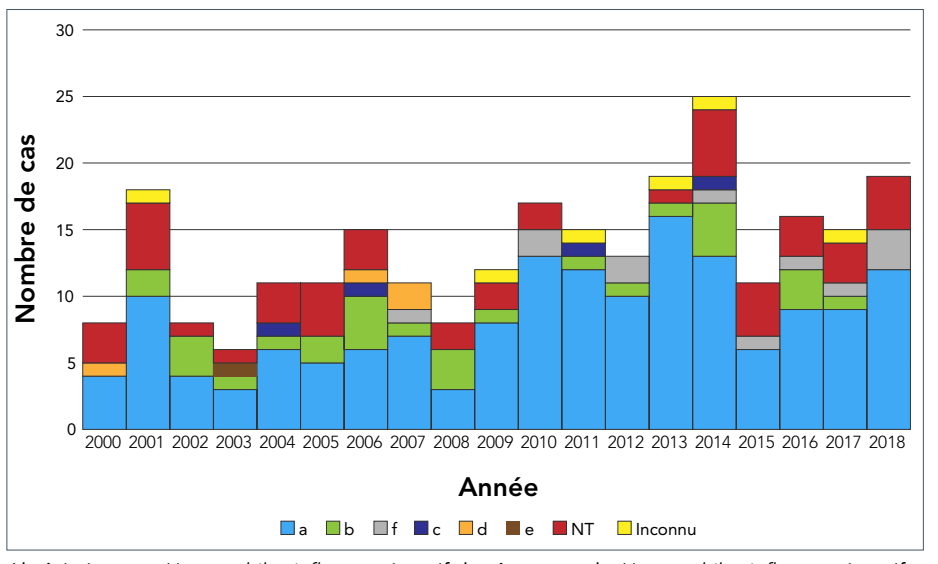

Abréviations : $a$, Haemophilus influenzae invasif de sérotype $a$; $b$, Haemophilus influenzae invasif de sérotype b; c, Haemophilus influenzae invasif de sérotype c; d, Haemophilus influenzae invasif de sérotype d; f, Haemophilus influenzae invasif de sérotype f; NT, souches non typables

$222(86,0 \%)$ ont été hospitalisés. Sur les 226 cas pour lesquels on disposait de renseignements sur les résultats, 19 décès $(8,4 \%)$ ont été signalés. Parmi ces 19 cas, 11 étaient de sérotype a, cinq étaient non typables, deux étaient de sérotype $f$ et un de sérotype $b$.

Aucune différence significative n'a été observée dans les taux d'hospitalisation et de mortalité des hommes et des femmes (tableau 1).

\section{Infection invasive à méningocoques}

Il n'y a pas eu de changement significatif du taux d'incidence de l'infection invasive à méningocoque (pour 100000 habitants) entre 2000 et 2018 ( $\beta=0,03 ; p=0,3$ ). Le taux d'incidence (pour 100000 habitants) était le plus élevé chez les nourrissons de moins d'un an $(29,57$; IC : 17,83-49,04) et les enfants de 1 à 4 ans $(3,99 ; I C: 2,00-7,98)$.

Sur les 31 cas d'infections invasives à méningocoque, 16 cas (52\%) étaient de sérogroupe $B$, sept (23\%) de sérogroupe $W$, quatre $(13 \%)$ de sérogroupe $C$, un $(3 \%)$ de sérogroupe $Y$ et trois (10\%) de sérogroupe inconnu (figure 5). Les sept cas de sérogroupe W sont tous survenus entre 2012 et 2018 , constituant $47 \%$ de tous les cas d'infections invasives à méningocoque au cours de cette période. Aucun cas de sérogroupe W n'a été signalé avant 2012.

L'âge médian des cas de sérogroupe $B$ était inférieur à un an, avec un intervalle de 0 à 30 ans; l'âge médian des cas de sérogroupe $W$ était inférieur à un an, avec un intervalle de 0 à 28 ans; et l'âge médian des cas de sérogroupe C était de 29,5 ans, avec un intervalle de 0 à 56 ans.

La différence des taux d'incidence annuels moyens entre les personnes d'origine autochtone et non autochtone n'était pas significative (tableau 1). Parmi les 30 cas pour lesquels on 
lesquels on disposait de renseignements sur la manifestation

Figure 5 : Répartition des cas d'infection invasive à méningocoques, par année et par sérogroupe, Nord du Canada, 2000 à 2018

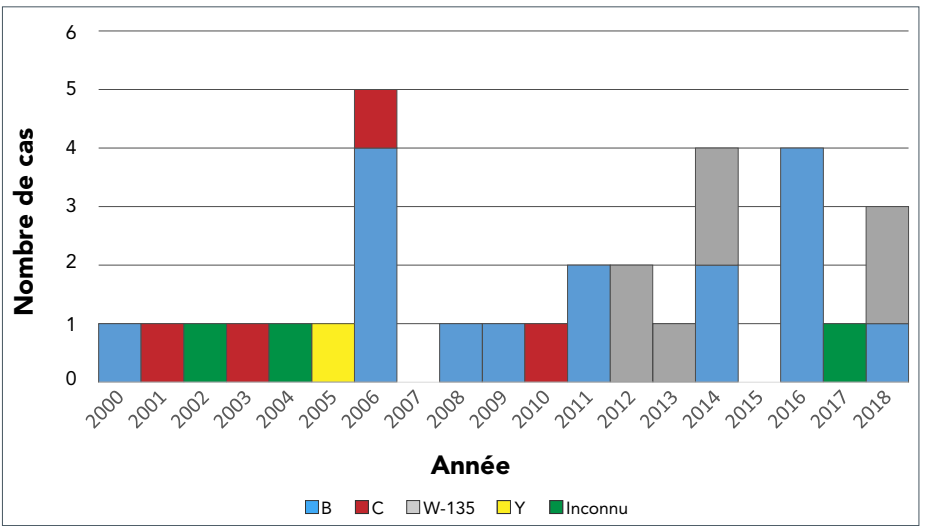

Abréviations : $B$, infection invasive à méningocoque de sérotype $B ; C$, infection invasive à méningocoque de sérotype $\mathrm{C} ; \mathrm{W}$, infection invasive à méningocoque $\mathrm{W} ; \mathrm{Y}$, infection invasive à méningocoque de sérotype $Y$

disposait de renseignements sur la manifestation clinique, la méningite ( $n=21 ; 70 \%)$ et la bactériémie ( $n=13 ; 43 \%)$ étaient les plus fréquemment rapportées et $90 \%$ ont été hospitalisés. Sur les 30 cas pour lesquels on dispose de renseignements sur la mortalité, quatre décès (TL : $13 \%$ ) ont été signalés.

Aucune différence significative n'a été observée dans les taux d'hospitalisation et de mortalité des hommes et des femmes (tableau 1).

\section{Infection invasive à streptocoque du groupe B}

II n'y a pas eu de changement significatif du taux d'incidence de streptocoques du groupe B entre 2000 et 2018 ( $\beta=-0,01$; $p=0,6$ ). Le taux d'incidence (pour 100000 habitants) était le plus élevé chez les nourrissons de moins d'un an $(46,16$; IC : 29,09-73,26) et les adultes de 60 ans et plus $(3,97$; IC : 2,07-7,64). La différence des taux d'incidence annuels moyens entre les personnes d'origine autochtone et non autochtone n'était pas significative (tableau 1).

Sur les 44 cas de streptocoques du groupe B, 16 cas (36\%) étaient néonatals et 28 cas (64\%) étaient non néonatals. Sur les 16 cas néonatals, 10 étaient des maladies à apparition précoce, cinq des maladies à apparition tardive et un était inconnu. Sur les sept cas néonatals pour lesquels on disposait de renseignements sur le sérotype, quatre étaient de sérotype III et les trois autres de sérotype la, lb et $\mathrm{V}$. Sur les 15 cas néonatals pour lesquels on disposait de renseignements sur la manifestation clinique, la bactériémie $(n=10)$ et la méningite $(n=6)$ étaient les plus fréquemment rapportées.

Parmi les 28 cas non néonatals, l'âge médian était de 55,5 ans (intervalle 0-88 ans). Parmi les 25 cas non néonatals avec renseignements sur le sérotype, 6 (24\%) étaient des sérotypes III et $\mathrm{V}$ chacun, trois (12\%) étaient des sérotypes la, lb et $\mathrm{VI}$ chacun, deux (8\%) étaient des sérotypes IV, et un était un sérotype II et un sérotype $\mathrm{VI}$ ( $4 \%$ chacun). Parmi les 27 cas non néonatals pour clinique, la bactériémie $(n=21 ; 78 \%)$, l'arthrite septique ( $n=6$, $22 \%$ ) et la cellulite ( $n=5,19 \%$ ) étaient les plus fréquemment signalées. Parmi tous les cas non néonatals, $93 \%$ ont été hospitalisés; aucun décès n'a été signalé.

Aucune différence significative n'a été observée entre les taux d'hospitalisation et de mortalité des hommes et des femmes (tableau 1).

\section{Discussion}

Dans ce rapport, nous décrivons l'épidémiologie des maladies bactériennes invasives dans le Nord du Canada de 1999 à 2018 et nous comparons les taux d'incidence dans cette région à ceux du reste du Canada. Les taux de la plupart des maladies invasives sont restés stables dans le temps, à l'exception de la pneumococcie invasive, qui a eu tendance à baisser, et de l'infection invasive à streptocoque du groupe A, qui a eu tendance à augmenter dans le temps. L'incidence de pneumococcies invasives, d'infections invasives à streptocoque du groupe $A$ et de $H$. influenzae sérotype $b$ était respectivement $2,8,3,2$ et 8,8 fois plus élevée dans le Nord du Canada que dans le reste du pays. Dans le nord du Canada, les taux d'incidence annuels moyens de pneumococcies invasives, d'infections invasives à streptocoque du groupe $\mathrm{A}$ et $\mathrm{d}^{\prime} \mathrm{H}$. influenzae étaient nettement plus élevés chez les personnes s'identifiant comme autochtones que chez celles d'origine non autochtone. Les taux $d$ 'incidence les plus élevés ont été enregistrés chez les très jeunes et les personnes âgées.

À l'instar de la population de la surveillance circumpolaire internationale de l'Alaska (communication personnelle, T. Zulz, 19 janvier 2021), dans le Nord du Canada, la pneumococcie invasive a présenté le fardeau le plus lourd de la maladie et a représenté plus de la moitié du total des cas signalés pendant la période d'étude. L'incidence d'infections invasives à streptocoque du groupe A dans les régions du Nord est en augmentation, une tendance qui a également été observée à l'échelle nationale, où le taux d'incidence a été multiplié par trois entre 2000 et 2018 (34), avec des foyers dans la communauté, les bases militaires et les refuges (35-38). H. influenzae à sérotype a représentait plus de $60 \%$ des cas et a augmenté de manière significative au fil des années. Il existe également une prévalence élevée $d^{\prime} H$. influenzae à sérotype a dans la population de l'Alaska (communication personnelle, T. Zulz, 19 janvier 2021). Selon les données recueillies auprès des sites américains de surveillance Active Bacterial Core, entre 2002-2008 et 2009-2015, la prévalence de la maladie d'H. influenzae à sérotype a a augmenté de $13 \%$ par an, avec une augmentation globale de $148 \%$ (39).

La charge de morbidité de l'infection invasive à méningocoque et du streptocoque du groupe $B$ est beaucoup plus faible que celle des trois autres maladies, et les taux d'infections invasives 
à méningocoque et du streptocoque du groupe $B$ n'ont pas changé de manière significative au cours de la période étudiée. Les résultats indiquent également qu'il n'y a pas de différence statistique entre les taux d'incidence normalisés selon l'âge de l'infection invasive à méningocoque pour le reste du Canada et le Nord du Canada.

Les taux de pneumococcie invasive ont considérablement diminué au fil des ans en raison d'une baisse significative des sérotypes spécifiques des vaccins Pneu-C-7 et Pneu-C-10, ce qui indique que les programmes de vaccins conjugués ont réussi à réduire la circulation des sérotypes contre lesquels ces vaccins protègent. II n'y a pas eu de diminution significative des sérotypes spécifiques du Pneu-C-13 au fil des ans. D'autres études sont nécessaires pour surveiller l'impact du vaccin Pneu-C-13 par rapport au vaccin Pneu-C-10, surtout à la suite du passage récent du calendrier de vaccination par le Pneu-C-13 à un calendrier mixte de vaccination par le Pneu-C-10 et le Pneu-C-13 dans les régions du Québec.

Les sérotypes non vaccinaux ont considérablement augmenté après l'introduction des programmes de vaccins conjugués. Le nombre de sérotypes spécifiques du Pneu-C-23 a également augmenté et le pourcentage élevé de cas d'infection postvaccinale indique que le vaccin Pneu-C-23 n'est pas aussi efficace pour prévenir la maladie dans la population de surveillance circumpolaire internationale de 65 ans et plus que les vaccins conjugués dans la population de surveillance circumpolaire internationale plus jeune.

Les taux $d^{\prime} H$. influenzae sérotype $b$ n'ont pas changé de façon significative dans le Nord du Canada au cours de cette période d'étude. Aucun cas d'infections invasives à méningocoque du sérogroupe $C$ n'a été signalé depuis 2010, ce qui pourrait être dû à la mise en œuvre du vaccin Men-C-C dans les programmes de vaccination systématique des enfants au milieu des années 2000 (40). Au cours des sept dernières années, les sérogroupes $B$ et $W$ ont été signalés exclusivement et la charge la plus élevée a été enregistrée chez les nourrissons de moins d'un an.

\section{Forces et faiblesses}

Le programme surveillance circumpolaire internationale est une source importante de renseignements épidémiologiques et liés aux laboratoires. II s'agit du seul système amélioré de surveillance des maladies bactériennes invasives qui fournit des renseignements sur l'ethnicité au Canada, ce qui signifie qu'il peut être utilisé pour surveiller l'épidémiologie des maladies bactériennes invasives chez les peuples autochtones du Nord du Canada.

La surveillance circumpolaire internationale est un système de surveillance passif et certains cas peuvent être manqués. Dans certaines collectivités, la prise d'antibiotiques peut avoir commencé avant le prélèvement des cultures. Les résultats sont instables en raison du petit nombre de cas et de la petite taille de la population; il convient donc de faire preuve de prudence dans l'interprétation des résultats. En raison de la déclaration incomplète des manifestations cliniques, des antécédents vaccinaux et des conditions et facteurs de risque sous-jacents, ces résultats peuvent être sous-estimés ou surestimés. En outre, tous les cas n'ont pas fait l'objet d'un sérotypage supplémentaire.

\section{Conclusion}

Le fardeau des maladies bactériennes invasives est plus lourd dans la population nordique du programme de surveillance circumpolaire internationale que dans le reste du Canada, en particulier dans la population autochtone. Nous disposons de peu de renseignements sur la santé de l'importante population autochtone du Nord du Canada, mais les disparités existantes en matière de santé doivent être surveillées et traitées. Une surveillance continue est nécessaire pour continuer à suivre les tendances de la maladie, soutenir les stratégies de prévention et de contrôle, et orienter les recommandations en matière d'immunisation.

\section{Déclaration des auteurs}

G. H. - Méthodologie, logiciel, analyse formelle, enquête, conservation des données, rédaction-version originale, rédaction-révision et édition, visualisation

I. M. - Méthodologie, analyse formelle, rédaction-version originale, rédaction-révision et édition

R. S. T. - Méthodologie, analyse formelle, rédaction-version originale, rédaction-révision et édition

W. H. D. - Méthodologie, analyse formelle, rédaction-version originale, rédaction-révision et édition

G. J. T. - Méthodologie, analyse formelle, rédaction-version originale, rédaction-révision et édition

Y. A. L. - Méthodologie, analyse formelle, rédaction--révision, édition et administration du projet

C. D. - Conceptualisation, écriture-révision et édition

F. R.-D. - Méthodologie et rédaction-révision et édition

S. G. S. - Conceptualisation, méthodologie, rédaction-révision et édition, administration du projet

\section{Intérêts concurrents}

Aucun.

\section{Remerciements}

Nous souhaitons remercier tous les membres du groupe de travail canadien sur les maladies bactériennes invasives du Système de surveillance circumpolaire internationale pour 
leur contribution inestimable à la surveillance circumpolaire internationale.

\section{Financement}

La participation du Canada à la surveillance circumpolaire internationale a été financée par l'Agence de santé publique du Canada.

\section{Références}

1. Parkinson AJ, Bruce MG, Zulz T, Parkinson A; International Circumpolar Surveillance Steering Committee. International Circumpolar Surveillance, an Arctic network for the surveillance of infectious diseases. Emerg Infect Dis 2008;14(1):18-24. DOI PubMed

2. Vaudry W, Talling D. Infection invasive à pneumocoques chez des enfants des Premières Nations du Nord de I'Alberta. Relevé des maladies transmissibles au Canada 2002;28(20):165-72. https://publications.gc.ca/collections/ Collection/H12-21-28-20.pdf

3. Christiansen J, Paulsen P, Ladefoged K. Invasive pneumococcal disease in Greenland. Int J Circumpolar Health 2004;63 Suppl 2:214-8. DOI PubMed

4. Singleton R, Hammitt L, Hennessy T, Bulkow L, DeByle C, Parkinson A, Cottle TE, Peters H, Butler JC. The Alaska Haemophilus influenzae type $b$ experience: lessons in controlling a vaccine-preventable disease. Pediatrics 2006;118(2):e421-9. DOl PubMed

5. Degani N, Navarro C, Deeks SL, Lovgren M. Invasive bacterial diseases in northern Canada. Emerg Infect Dis 2008;14(1):34-40. DOl PubMed

6. Bruce MG, Deeks SL, Zulz T, Navarro C, Palacios C, Case C, Hemsley C, Hennessy T, Corriveau A, Larke B, Sobel I, Lovgren M, Debyle C, Tsang R, Parkinson AJ. Epidemiology of Haemophilus influenzae serotype a, North American Arctic, 2000-2005. Emerg Infect Dis 2008;14(1):48-55. DOI PubMed

7. Li YA, Martin I, Tsang R, Squires SG, Demczuk W, Desai S. Maladies bactériennes invasives dans le Nord du Canada de 2006 à 2013. Relevé des maladies transmissibles au Canada 2016;42:84-91. DOI

8. Statistique Canada. Tableaux de données, Recensement 2016. Catalogue no. 98-400-X2016156. Ottawa (ON) : Statistique Canada; (accédé 2020-01-31). https://www12. statcan.gc.ca/census-recensement/2016/dp-pd/dt-td/Rpeng.cfm?TABID $=2 \&$ Lang $=E \& A P A T H=3 \& D E T A I L=0 \& D I M=0$ $\& F L=A \& F R E E=0 \& G C=0 \& G I D=1257309 \& G K=0 \& G R P=1 \& P I$ $\mathrm{D}=110511 \& \mathrm{PRID}=10 \& \mathrm{PTYPE}=109445 \& \mathrm{~S}=0 \& \mathrm{SHOWALL}=0 \&$ SUB $=0 \&$ Temporal $=2017 \&$ THEME $=122 \& V I D=0 \& V N A M E E=\&$ $\mathrm{VNAMEF}=\& \mathrm{D} 1=0 \& \mathrm{D} 2=0 \& \mathrm{D} 3=0 \& \mathrm{D} 4=0 \& \mathrm{D} 5=0 \& \mathrm{D} 6=0$
9. Agence de la santé publique du Canada. Guide canadien d'immunisation : Introduction [Internet]. Ottawa (ON) : ASPC; 2020 (accédé 2020-06-30). https://www.canada.ca/ $\mathrm{fr} /$ sante-publique/services/guide-canadien-immunisation/ introduction.html

10. Déclaration d'un comité consultatif (DCC) Comité consultatif national de l'immunisation (CCNI). Mise à jour sur les pneumococcies invasives chez les enfants et I'utilisation recommandée du vaccin conjugué contre le pneumocoque. Relevé des maladies transmissibles au Canada 2010;36(ACS-3):1-30. https://www.canada.ca/ content/dam/phac-aspc/migration/phac-aspc/publicat/ccdrrmtc/10pdf/36-acs-3.pdf

11. Papadatou I, Spoulou V. Pneumococcal vaccination in high-risk individuals: are we doing it right? Clin Vaccine Immunol 2016;23(5):388-95. DOI PubMed

12. Government of Newfoundland and Labrador. Newfoundland and Labrador immunization manual: section 2: routine immunization schedules. St John's (NL): Government of Newfoundland and Labrador; 2019.

13. Government of Newfoundland and Labrador. Newfoundland and Labrador immunization manual: section 5: immunization programs for high risk groups. St John's (NL): Government of Newfoundland and Labrador; 2019.

14. Government of Yukon. Yukon immunization program: section 8 - biological products. Whitehorse (YU): Government of Yukon; 2019.

15. Government of Northwest Territories. NWT immunization schedule: publicly funded vaccines for infants and children. Yellowknife (NT): Government of Northwest Territories; 2018.

16. Chief Public Health Office. Routine immunization schedule 2010: adults previously immunized. Iqaluit (NU): Government of Nunavut; 2010.

17. Gouvernement du Quebec. Vaccin polysaccharidique contre le pneumocoque. Québec ( $\mathrm{QC}$ ) : Gouvernement du Quebec; 2017 (accédé 2020-05-31). https://www.quebec.ca/sante/ conseils-et-prevention/vaccination/vaccin-pneumocoquepolysaccharidique

18. Agence de la santé publique du Canada. Défi nitions nosologiques des maladies transmissibles faisant l'objet d'une surveillance nationale. Relevé des maladies transmissibles au Canada 2009;35 Suppl 2:1-134. https://www.canada.ca/content/dam/phac-aspc/migration/ phac-aspc/publicat/ccdr-rmtc/09pdf/35s2-fra.pdf

19. Austrian R. The quellung reaction, a neglected microbiologic technique. Mt Sinai J Med 1976;43(6):699-709. PubMed

20. Tyrrell GJ, Lovgren M, Kress B, Grimsrud K. Invasive group A streptococcal disease in Alberta, Canada (2000 to 2002). J Clin Microbiol 2005;43(4):1678-83. DOI PubMed

21. Altschul SF, Gish W, Miller W, Myers EW, Lipman DJ. Basic local alignment search tool. J Mol Biol 1990;215(3):403-10. DOI PubMed 
22. Centers for Disease Control and Prevention. Streptococcus Laboratory. Protocol for emm typing. Atlanta (GA): CDC; 2018 (accédé 2020-03-31). https://www.cdc.gov/streplab/ groupa-strep/emm-typing-protocol.html

23. Slotved HC, Elliott J, Thompson T, Konradsen HB. Latex assay for serotyping of group B Streptococcus isolates. J Clin Microbiol 2003;41(9):4445-7. DOI PubMed

24. Ledeboer NA, Doern GV. Haemophilus. In: Versalovic J, Carroll KC, Jorgensen JH, Funke G, Landry ML, Warnock DW, editors. Manual of clinical microbiology. $10^{\text {th }}$ ed. Washington (DC): ASM Press; 2011. p. 585-602.

25. Falla TJ, Crook DW, Brophy LN, Maskell D, Kroll JS, Moxon ER. PCR for capsular typing of Haemophilus influenzae. J Clin Microbiol 1994;32(10):2382-6. DOI PubMed

26. Riou JY, Guibourdenche M. Laboratory methods: Neisseria and Branhamella. Paris (FR): Institut Pasteur; 1992.

27. Clinical Laboratory Standards Institute. CLSI M100-S26: Performance standards for antimicrobial susceptibility testing, 26 ${ }^{\text {th }}$ ed. Wayne (PA): CLSI; 2016.

28. Statistique Canada. Peuples autochtones du Canada : Faits saillants en tableaux, Recensement de 2001. Tableau : 97F0024X2001007. Ottawa (ON) : Statistique Canada; (accédé 2020-01-31). https://www150.statcan.gc.ca/n1/fr/ catalogue/97F0024X2001007

29. Statistique Canada. Enquête nationale auprès des ménages de 2011. Tableaux de données. No au catalogue : 99-011X2011034. Ottawa (ON) : Statistique Canada; (modifié 2016-01-07; accédé 2020-01-31). https://www12.statcan. gc.ca/nhs-enm/2011/dp-pd/dt-td/Ap-eng.cfm?LANG=E\&AP $\mathrm{ATH}=3 \& \mathrm{DETAIL}=0 \& \mathrm{DIM}=0 \& \mathrm{FL}=\mathrm{A} \& \mathrm{FREE}=0 \& \mathrm{GC}=0 \& \mathrm{GID}=0$ $\& G K=0 \& G R P=0 \& P I D=107504 \& P R I D=0 \& P T Y P E=105277 \& S$ $=0 \&$ SHOWALL $=0 \& S U B=0 \&$ Temporal $=2013 \&$ THEME $=94 \& \mathrm{~V}$ $\mathrm{ID}=0 \& \mathrm{VNAMEE}=\& \mathrm{VNAMEF}=$

30. Statistique Canada. 2006 Profil - Peuples autochtones, pour le Canada, les provinces, les territoires, les divisions de recensement et les subdivisions de recensement, Recensement de 2006. Recensement de la population de 2006, produit numéro 94-578-XCB2006001. Ottawa (ON) : Statistique Canada; (modifié 2015-02-09; accédé 2020-01-31). https://www12.statcan.gc.ca/francais/census01/ products/highlight/Aboriginal/Index.cfm?Lang=F

31. Statistique Canada. Tableaux de données, Recensement de 1996. No catalogue. 95F0251XDB96001. Ottawa (ON) : Statistique Canada; (modifié 2019-06-04; accédé 2020-01-31). https://www12.statcan.gc.ca/francais/ census96/data/tables/Rp-fra.cfm?LANG $=F \& A P A T H=3 \& D$ $E T A I L=1 \& D I M=0 \& F L=A \& F R E E=1 \& G C=0 \& G I D=0 \& G K=0$ $\& G R P=1 \& P I D=15504 \& P R I D=0 \& P T Y P E=89103 \& S=0 \& S H$ OWALL $=$ No\&SUB $=0 \&$ Temporal $=1996 \&$ THEME $=7 \& \mathrm{VID}=0$ $\& \mathrm{VNAMEE}=\& \mathrm{VNAMEF}=$
32. Centre for Population Health Data. 2011 Population Weights. Ottawa (ON): Statistics Canada; 2020.

33. Fay MP, Feuer EJ. Confidence intervals for directly standardized rates: a method based on the gamma distribution. Stat Med 1997;16(7):791-801. PubMed

34. Agence de la santé publique du Canada. Graphiques pré-construits de maladies à déclaration obligatoire. Ottawa (ON) : ASPC; 2021 (accédé 2021-02-28). https://maladies. canada.ca/declaration-obligatoire/graphiques-pre-construits

35. Loewen K, Bocking N, Matsumoto CL, Kirlew M, Kelly L. Epidemiologic features of invasive group A Streptococcus infection in a rural hospital: 6-year retrospective report and literature review. Can J Rural Med 2017;22(4):131-8. PubMed

36. Athey TB, Teatero $S$, Sieswerda LE, Gubbay JB Marchand-Austin A, Li A, Wasserscheid J, Dewar K, McGeer A, Williams D, Fittipaldi N. High incidence of invasive group A Streptococcus disease caused by strains of uncommon emm types in Thunder Bay, Ontario, Canada. J Clin Microbiol 2016;54(1):83-92. DOI PubMed

37. Hammond-Collins K, Strauss B, Barnes K, Demczuk W, Domingo MC, Lamontagne MC, Lu D, Martin I, Tepper M. Group A Streptococcus Outbreak in a Canadian Armed Forces Training Facility. Mil Med 2019;184(3-4):e197-204. DOI PubMed

38. Dickson C. 2021 International Circumpolar Surveillance Invasive Bacterial Disease Working Group meeting. Updating public health guidelines for invasive group A streptococcus. Forthcoming, 2021.

39. Soeters HM, Blain A, Pondo T, Doman B, Farley MM, Harrison LH, Lynfield R, Miller L, Petit S, Reingold A, Schaffner W, Thomas A, Zansky SM, Wang X, Briere EC. Current epidemiology and trends in invasive Haemophilus influenzae disease-United States, 2009-2015. Clin Infect Dis 2018;67(6):881-9. DOI PubMed

40. Comité consultatif national de l'immunisation (CCNI). Recommandations concernant l'administration des vaccins conjugués contre le méningocoque de sérogroupe $C$ aux nourrissons. Une déclaration d'un comité consultatif (DCC). Relevé des maladies transmissibles au Canada 2007;33(ACS-11):1-12. https://www.canada.ca/fr/santepublique/services/rapports-publications/releve-maladiestransmissibles-canada-rmtc/numero-mensuel/2007-33/ preambule.html 